\title{
Anxiety and the differential adaptation hypothesis
}

\author{
Alfred Castaneda \\ HUNTER COLEGE and MOUNT SINAI HOSPITAL
}

\begin{abstract}
Spence's suggestion that high anxious individuals exhibit relatively less adaptation to threat or aversive forms of stimulation than low anxious individuals is examined and one of its implications for research is described. His suggestion is termed the differential adaptation hypothesis in the present paper.
\end{abstract}

Reviews of research with the Manifest Anxiety Scale (MAS) by Spence (1956) and Taylor (1956a) indicate that although MAS scores are assumed to reflect individual differences in the emotional state of $\mathrm{S}$, such differences may not be characteristic of individuals under all circumstances. Consequently, these writers have evolved two alternative hypotheses concerning the conditions under which differences in emotionality between high anxious (HA) and low anxious (LA) Ss may be observed: (1) One is the chronic hypothesis; HA Ss may react emotionally in a chronic manner to all situations, whether stressful or not. (2) The other is the reactive hypothesis; the tendency for HA Ss to react with greater emotionality than LA Ss is a positive function of the degree to which the situation is stressful.

Although Spence did not elaborate its implications, he did propose a third hypothesis, namely, that HA Ss would "... show relatively less adaptation to situations containing a threat or some degree of noxiousness than do subjects who score at the low end of the scale." (Spence, 1956, p. 183). This third alternative notion we shall term the differential adaptation hypothesis. The following sections examine one of its implications in detail.

It is generally assumed that the sensory consequences associated with aversive forms of stimulation, such as the UCS in classical defense conditioning, evoke an internal, emotional response coincidentally with other, overt, unconditioned responses. Early writers in the field of conditioning suggested that the affective aspects of the noxious UCS, i.e., its emotion-evoking property, might function as a source of primary motivation in the conditioning situation. This assumption has been articulated in the writings of Mowrer and Miller who, respectively, designated the conditioned form of this hypothetical, emo- tional response, anxiety, fear.

Several studies have assessed the hypothesis that this internal, emotional response, as in the case of the observable UCRs, also possesses the property of adaptation, i.e., decrements in its vigor produced by its repeated re-arousal. McDonald (1956) for example, showed that the conditioning performance of Ss given adaptation training with the UCS alone prior to its being paired in the conditioning procedure with the CS was lower than that of Ss given conditioning training without such prior adaptation experience. McDonald assumed that the emotional response is one of the factors contributing to drive strength in defense conditioning. Her results are consistent with the hypothesis that the emotional response was reduced in strength as a function of the adaptation experience. This adaptation effect, i.e., the reduction in the level of performance in conditioning has also been recently observed by Taylor (1956b).

Following Spence's original suggestion that one of the differences between HA and LA Ss is in the adaptive property of the emotional response, one implication of these two studies is that the adaptation effect differentiates between them. Fig. 1 schematically depicts the major assumptions underlying this hypothesis. The ordinate represents some measure of the magnitude or strength of the emotional response, the abscissa, the number of presentations of the aversive stimulus to be employed as the UCS in sub-

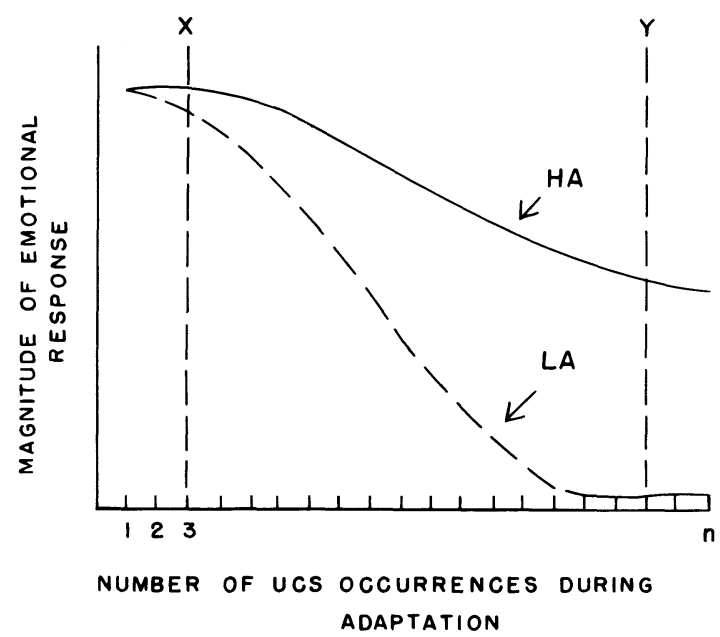


sequent conditioning training. The unbroken line represents decrements in the magnitude of the hypothetical, emotional response of the HA Ss, the broken line, that of the LA Ss. Note that although the emotional response exhibits adaptation as a function of its repeated evocation for both HA and LA Ss, the rate at which this effect takes place is different for the two. That is, the emotional response of the HA Ss is assumed to adapt at a relatively slower rate than that of the LA Ss. If the strength of the emotional response is a determinant of drive strength, then both groups would experience a decrement in drive, but the difference in the level of the drive between the two groups would be increased in favor of the HA Ss.

The major implication of this assumption for conditioning performance is indicated by the two vertical lines, " $\mathrm{X}$ " and " $\mathrm{Y}$ ". $\mathrm{X}$ bisects the $\mathrm{ab-}$ scissa at point " 3 " and $\mathrm{Y}$ bisects at a larger, though unspecified point. Assuming that point " $X$ " represents the effects of those few adaptation trials with the UCS which is standard procedure in most eyelid conditioning experiments, while the point at which point " $Y$ " bisects the two curves represents the level of emotional responsiveness to the UCS after some greater amount of adaptation training, it would be expected that differences in the level of conditioning between HA and LA Ss would depend on the extent of adaptation training. That is, while the level of conditioning would be expected to be lower after adaptation training for both groups, the differ- ence between HA and LA Ss would be expected to be larger the greater the amount of prior adaption training. More specifically, the HA and LA Ss given the lesser amount of adaptation training, i.e., at point ' $X$ ", would be expected to exhibit a generally higher level of conditioning performance than the two groups given the greater amount. More pertinent, however, is the implication that the absolute difference in the level of conditioning performance between HA and LASs is expected to be larger the greater the amount of adaptation training. Re-phrased in terms of an experimental design, the present differential adaptation hypothesis would be tested by means of an interaction in conditioning performance between the two major factors, anxiety level and amount of prior adaptation training.

\section{References}

MACDONALD, ANNETTE. The effect of adaptation to the unconditioned stimulus upon the formation of conditioned avoidance responses. J. exp. Psychol., 1946, $36,1-12$.

SPENCE, K. W. Behavior theory and conditioning. New Haven: Yale University Press, 1956.

TAYLOR, JANET A. Drive theory and manifest anxiety. Psychol. Bull., 1956a, 53, 303-320.

TAYLOR, JANET A. Level of conditioning and intensity of the adaptation stimulus. J. exp. Psychol., 1956b, 51, 127-130.

\section{Acknowledgment}

The preparation of this report was facilitated by a NIMH Grant (M-4240-03) to the author. 